\title{
Ultrathin graphitic structures and carbon nanotubes in a purified synthetic graphite
}

Article

Accepted Version

Final version includes peer review changes

Harris, P. J. F. (2009) Ultrathin graphitic structures and carbon nanotubes in a purified synthetic graphite. Journal of Physics: Condensed Matter, 21 (35). 355009. ISSN 1361-648X doi: https://doi.org/10.1088/0953-8984/21/35/355009 Available at https://centaur.reading.ac.uk/24751/

It is advisable to refer to the publisher's version if you intend to cite from the work. See Guidance on citing.

To link to this article DOI: http://dx.doi.org/10.1088/0953-8984/21/35/355009

Publisher: Institute of Physics Publishing

All outputs in CentAUR are protected by Intellectual Property Rights law, including copyright law. Copyright and IPR is retained by the creators or other copyright holders. Terms and conditions for use of this material are defined in the End User Agreement.

\section{www.reading.ac.uk/centaur}

\section{CentAUR}

Central Archive at the University of Reading

Reading's research outputs online 


\section{Ultrathin graphitic structures and carbon nanotubes in a purified synthetic graphite}

Peter J.F. Harris

Centre for Advanced Microscopy, J.J. Thomson Physical Laboratory, University of Reading, Whiteknights, Reading RG6 6AF, UK.

E-mail: p.j.f.harris@reading.ac.uk

\section{Abstract}

A new class of carbon structure is reported, which consists of microscale graphitic shells bounded by curved and faceted planes containing 2 - 5 layers. These structures were originally found in a commercial graphite produced by the Acheson process, followed by a purification treatment. The particles, which could be several $100 \mathrm{~s}$ of $\mathrm{nm}$ in size, were frequently decorated with nano-scale carbon particles, or short nanotubes. In some cases, nanotubes were found to be seamlessly connected to the thin shells, indicating that the formation of the shells and the nanotubes is intimately connected. The structures are believed to form during a purification process which involves passing an electric current through the graphite in the presence of a reactive gas. In support of this, it is shown that similar particles can be produced in a standard carbon arc apparatus. With their extremely thin graphene walls and high surface areas the new structures may have a range of useful properties.

\section{Introduction}

Carbon is a material which continues to produce surprises. After the discovery of fullerenes ${ }^{1}$ and nanotubes ${ }^{2,3}$, the most recent form of carbon to be isolated, graphene ${ }^{4}$, is producing intense interest. Much of this interest has focussed on graphene's unique electronic characteristics ${ }^{5}$, but a range of other interesting properties have also been demonstrated ${ }^{6,7}$. This paper describes another new carbon material consisting of curved and faceted graphitic 
structures with ultrathin walls. These particles were initially found in commercial graphite furnace tubes used for atomic absorption spectroscopy. The discovery was accidental, and occurred during a programme of research into non-graphitizing carbons. The present author has a long-standing interest in the effect of heat-treatments on these carbons ${ }^{8-10}$. In order to heat the carbons to very high temperatures (up to $3000^{\circ} \mathrm{C}$ ), a graphite furnace attached to an atomic absorption spectrometer was used. When the heattreated carbons were examined, it was found that they sometimes contained the unusual graphitic structures and nanoparticles. At first it was thought that these were produced by the heat-treatment, but it was soon established that they originated in the graphite furnace tubes used to contain the samples. It is believed that they form during a purification process which involves passing an electric current through the graphite in the presence of a reactive gas. In order to test this idea, an initial examination was made of carbon from graphite rods through which a current had been passed in a commercial arcevaporation unit.

The new carbon structures might find applications in a wide variety of areas. For example, their extremely large surface-to-volume ratio and high conductivity suggest that they may be useful in sensors or electrical devices. They may also have potential in catalysis or as hydrogen storage materials.

\section{Experimental}

The graphite furnace tubes from which the novel carbon material was obtained were Perkin Elmer Standard Tubes (uncoated). These are small tubes, $28 \mathrm{~mm}$ long, with an outer diameter of $8 \mathrm{~mm}$, made from extremely pure graphite. To prepare specimens for transmission electron microscopy, carbon was gently scraped from the surface of the tubes, dispersed in isopropanol and then pipetted onto holey carbon TEM films.

As well as carbon from the graphite furnace tubes, a preliminary examination was made of carbon from graphite rods which had been used in an arcevaporation unit. The apparatus used was an Edwards S150B, a standard unit for coating specimens for scanning electron microscopy. In this unit, the 
electrodes are $3 \mathrm{~mm}$ graphite rods, one of which is sharpened to a point and held in contact with the other electrode with a spring mechanism. Discharge is carried out at a voltage of $10 \mathrm{~V}$, with a current of approximately $20 \mathrm{~A}$. The chamber is pumped by a rotary pump, giving a vacuum of approximately 0.1 Torr. Following arcing, carbon was scraped from the surface of the graphite rods and then prepared for TEM in the way described above. TEM examination was carried out in a Philips CM20, with a point resolution of $0.27 \mathrm{~nm}$, and a JEOL 2010, with a point resolution of $0.19 \mathrm{~nm}$, both operated at an accelerating voltage of $200 \mathrm{kV}$. Images were recorded photographically.

\section{Results}

When examined at moderate magnification it was clear that the samples obtained from the AAS furnace tubes contained two distinct types of material. Some of the carbon was in the form of "normal" graphite, i.e. flat crystallites, typically $1-5 \mu \mathrm{m}$ in diameter, containing up to 100 layers. However, a significant proportion of the samples consisted of shell-like structures with rounded and faceted shapes. Figure 1 (a) shows a typical region at low magnification, while Fig. 1 (b) shows a cluster of the hollow graphitic structures. In some cases these had outlines reminiscent of multiwalled carbon nanotubes (MWNTs), or carbon "nanohorns" ${ }^{11,12}$, but were generally far larger in size. Whereas MWNTs and nanohorns typically have outer diameters in the range 5 $-10 \mathrm{~nm}$, these new structures could be several 100s of $\mathrm{nm}$ in size. In some samples, the shell-like structures made up more than $50 \%$ of the material examined. An image of a faceted graphitic shell, with walls just 2 layers thick, is shown in Fig. 2. Bilayer microparticles of this kind were commonly seen, as were particles with $3-5$ layers, but single layer areas were very rare. The particle in Fig. 2 apparently has the form of a cone attached to an incomplete cylindrical region. The 3-dimensional shape of the particle is believed to be due to the presence of pentagonal rings, which introduce curvature into a graphene network. Support for this idea comes from an analysis of the angles which the facets make with each other. The angle of the apex labelled " $A$ " in Fig. 2 is exactly $60^{\circ}$, which is the angle produced by introducing 3 pentagons into a hexagonal network (see ref. [3], p121). The angles at "B" and "C" are 
approximately $150^{\circ}$. This is close to the angle observed in certain MWNT caps, where a conical region joins the cylindrical part of the tube (see ref. [3], p128).

It was often found that the shell-like structures were decorated with nanotubes and nanoparticles, as in the example shown in Fig. 3 (a). Most of the MWNTs observed here tended to be shorter than those grown in an arc, often being less than $100 \mathrm{~nm}$ in length. The presence of sharp bends in some of the tubes, as in Fig. 3 (a), and other discontinuities, indicated that they contained a relatively high concentration of defects. However, a few quite perfect tubes were found which were several 100 s of $\mathrm{nm}$ in length. Sometimes, nanotubes were found to be actually connected to the graphitic shells, as in Fig. 3 (b), indicating that the formation processes of the shells and the nanotubes are closely associated. The overall shape of the structure in Fig. 3 (b) appears to approximate to that of a funnel. The connection between the tube and the shell region here may involve 7-membered rings. Junctions of this kind between a nanotube and a graphene shell have not been previously reported, although junctions between nanotubes have been produced inside a TEM (e.g. refs. 13, 14).

Carbon from the graphite rods which had been used in the arc-evaporation unit was generally similar in appearance to that from the AAS tubes, although with an apparently higher proportion of nanotubes and nanoparticles. A typical region is shown at moderate magnification in Fig. 4(a). As can be seen, a large number of tube-like structures are present, supported on, or attached to, thin graphitic regions. Figure 4(b) is a higher magnification image of some of the thin regions. The structure on the left of the image resembles a rather imperfect MWNT cap, but with a much larger internal cavity.

\section{Discussion}

The graphite used for the AAS furnace tubes is prepared by the Acheson process, which is the standard method for producing synthetic graphite, followed by purification. It seems unlikely that the unusual graphitic structures 
form during the Acheson process, because they are not seen in other graphites produced by this method. It seems much more likely that they have formed during the purification treatment, which involves passing an electric current through the graphite in the presence of a reactive gas. This is a wellestablished technique for obtaining ultra-pure graphite ${ }^{15}$.

This idea that the thin graphite shells are produced by passing an electrical current through graphite is strongly supported by the observation that such structures are also found in carbon from graphite rods used for arcevaporation. It should be noted that the arc-evaporation in the current work was carried out under rather different conditions to those used in the classic lijima-Ebbesen-Ajayan synthesis of nanotubes ${ }^{2,16}$. In order to produce nanotubes in high yield, helium is introduced into the chamber, typically to a pressure of 500 Torr, and the graphite electrodes are usually held a short distance apart during arcing, rather than being kept in contact. Under these conditions, a hard cylindrical deposit forms on the cathodic rod, inside which are found the nanotubes. In the present work the arc-evaporator was operated in its normal mode, i.e. in a vacuum of approximately 0.1 Torr and with the electrodes in contact. This results in evaporation of carbon from the region near to the point of contact of the rods, but no deposit formation.

The growth mechanism of the thin graphitic shells is not known at present, but seems to be intimately related to the formation of the multiwalled nanoparticles and nanotubes which decorate the surfaces of these structures. Unfortunately the formation of carbon nanotubes themselves in the arc is very poorly understood ${ }^{3}$. One view, put forward by the present author ${ }^{17}$, is that nanotube growth is a solid state process in which disordered carbon which condenses onto the cathode is transformed into multiwalled nanotubes as a result of the high temperature and current it experiences in the arc. In the present case, the starting material is graphite rather than disordered carbon and the effect of arcing is apparently to convert much of the graphite into large hollow structures rather than nanotubes. Understanding the reasons for this, and the mechanism of the process will require further work, and such studies are now underway. 
When considering the effect on an electric current on the structure of graphite, it is interesting to note a recent paper by Jia et al. ${ }^{18}$. This described in situ Joule heating (i.e. heating by the passage of an electric current) of graphite "nanoribbons" inside a TEM. The structures produced by this treatment resemble some of those observed in the present study, although they tended to be single-layer structures rather than multilayer. The authors interpreted these structures in terms of zigzag and armchair edges of flat graphene. It is possible, however, that they were seeing hollow 3-dimensional structures like those described here.

As suggested in the introduction to this paper, the new hollow graphitic particles might be expected to have many interesting properties. One possible area might be in gas sensing, since both graphene ${ }^{19}$ and nanostructured graphite ${ }^{20}$ have been shown to have extremely sensitive sensing properties. Another potential application might be hydrogen storage, since forms of carbon rather similar to those described here have been shown to have $\mathrm{H}_{2}$ storage capabilities. Thus, Orimo et al. reported in 1999 that "nanostructured graphite" prepared by mechanical milling of synthetic graphite could have high $\mathrm{H}_{2}$ uptakes ${ }^{21}$. Carbon nanohorns have also been shown to have potentially useful $\mathrm{H}_{2}$ storage properties ${ }^{22}$. The new structures may also have useful electrical properties. Recently, it has been demonstrated that graphene can be used in electrochemical double layer capacitors ${ }^{23}$. The high surface area graphitic shells reported here might actually have advantages over conventional "flat" graphene in this application. The flat graphene particles would have a tendency to clump together, while the faceted shells would be more likely to retain their surface area. Finally the graphitic shells, with their highly perfect structures, might be expected to have excellent mechanical properties, and therefore be useful components of composite materials.

\section{Acknowledgement}

I thank Kazu Suenaga for discussions. 


\section{References}

[1] Kroto H W, Heath J R, O' Brien S C, Curl R F and Smalley R E 1985 Nature 318162

[2] lijima S 1991 Nature 35456

[3] Harris P J F 2009 Carbon Nanotube Science (Cambridge: Cambridge University Press)

[4] Novoselov K S, Geim A K, Morozov S V, Jiang D, Zhang Y, Dubonos S V, Grigorieva I V and Firsov A A 2004 Science 306666

[5] Neto A H C, Guinea F, Peres N M R, Novoselov K S and Geim A K 2009 Reviews of Modern Physics 81, 109

[6] Geim A K and Novoselov K S 2007 Nature Materials 6183

[7] Geim A K 2009 Science 3241530

[8] Harris P J F and Tsang S C 1997 Phil. Mag. A 76667

[9] Harris P J F 1997 Internat. Mater. Rev. 42206

[10] Harris P J F 2005 Crit. Rev. Sol. State Mat. Sci. 30235

[11] Harris P J F, Tsang S C, Claridge J B and Green M L H 1994 J Chem Soc, Faraday Trans 902799

[12] lijima S, Yudasaka M, Yamada R, Bandow S, Suenaga K, Kokai F, and Takahashi K 1999 Chem. Phys. Lett. 309165

[13] Terrones M, Banhart F, Grobert N, Charlier J C, Terrones H, Ajayan PM 2002 Phys. Rev. Lett. 89075505

[14] Jin C H, Suenaga K, lijima S 2008 Nature Nanotechnology 317

[15] Matsumoto T and Hoshikawa T 1995 United States Patent 5,419,889

[16] Ebbesen T W and Ajayan P M 1992 Nature 358220

[17] Harris P J F 2007 Carbon 45229

[18] Jia X, Hofmann M, Meunier V, Sumpter B G, Campos-Delgado J, RomoHerrera J M, Son H, Hsieh YP, Reina A, Kong J, Terrones M and Dresselhaus M S 2009 Science 3231701

[19] Schedin F, Geim A K, Morozov S V, Hill E W, Blake P, Katsnelson M I and Novoselov KS 2007 Nature Materials 6652 
[20] Qazi M, Vogt T and Koley G 2007 Appl. Phys. Lett. 91233101

[21] Orimo S, Majer G, Fukunaga T, Züttel A, Schlapbach L and Fujii H 1999 Appl. Phys. Lett. 753093

[22] Tanaka H, Kanoh H, El-Merraoui M, Steele W A, Yudasaka M, lijima S and Kaneko K 2004 J. Phys. Chem. B 10817457

[23] Stoller M D, Park S J, Zhu Y W, An J H and Ruoff R S 2008 Nano Lett. 8 3498

Figure Captions

Figure 1 (a) Low magnification micrograph of carbon from AAS tube showing region with thin, hollow, graphitic structures. A region of "normal" graphite can be seen at bottom right. (b) Higher magnification micrograph of another region with hollow structures.

Figure 2 Large hollow structure with bilayer graphene walls, found in carbon from AAS tube. The faceting at points " $\mathrm{A}$ ", " $\mathrm{B}$ " and " $\mathrm{C}$ " is due to the presence of pentagons.

Figure 3 Nanostructures found in carbon from AAS tube. (a) Multiwalled carbon nanotubes and nanoparticles supported on bilayer graphene shell, (b) bilayer nanotube directly attached to shell structure.

Figure 4 Structures found in carbon from graphite rods used for arcevaporation. (a) General region, with nanotubes and nanoparticles supported on thin graphitic structures, (b) higher magnification image showing shell-like structures. 

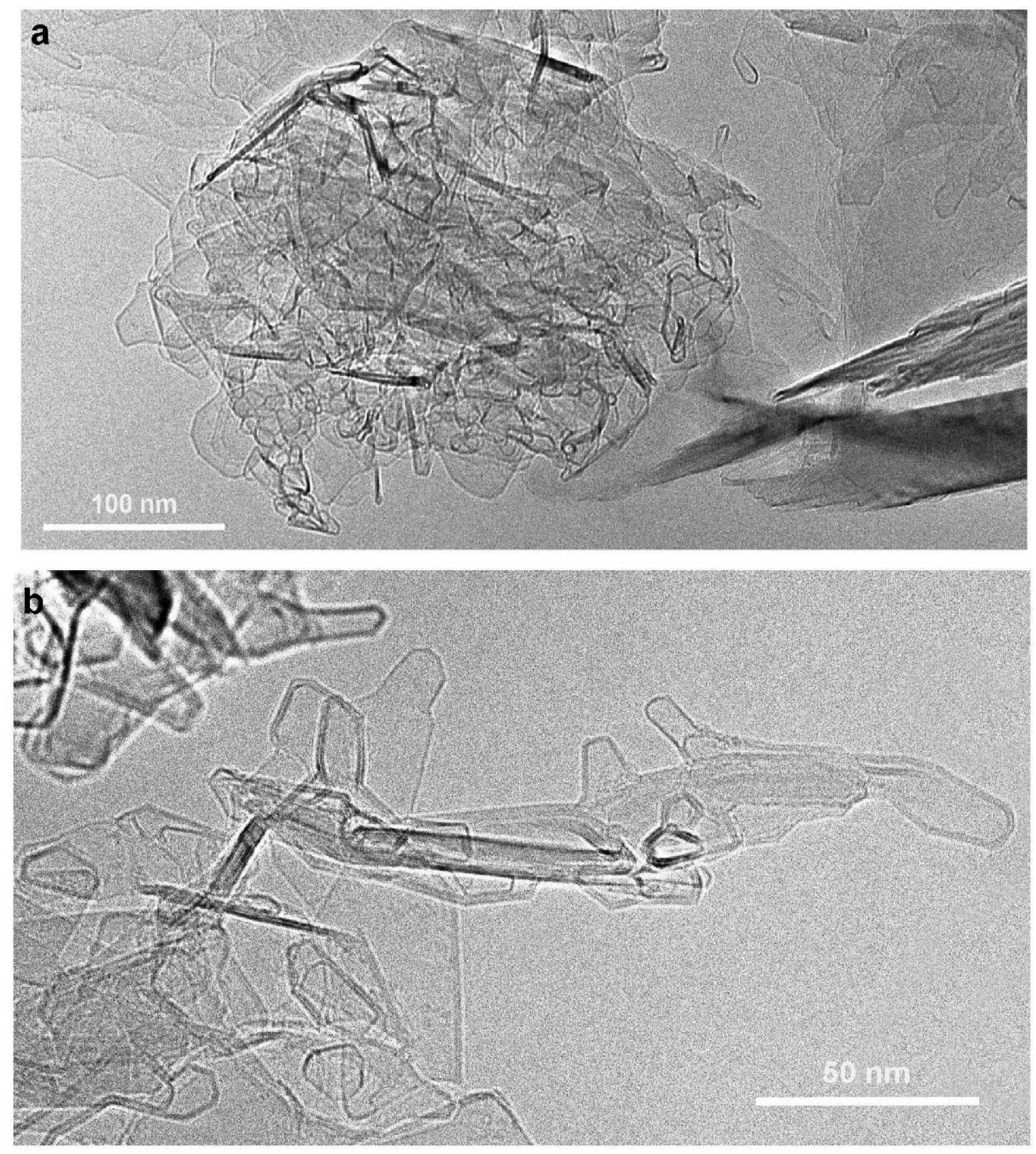

Figure 1 (Harris Fig 1.jpg) 


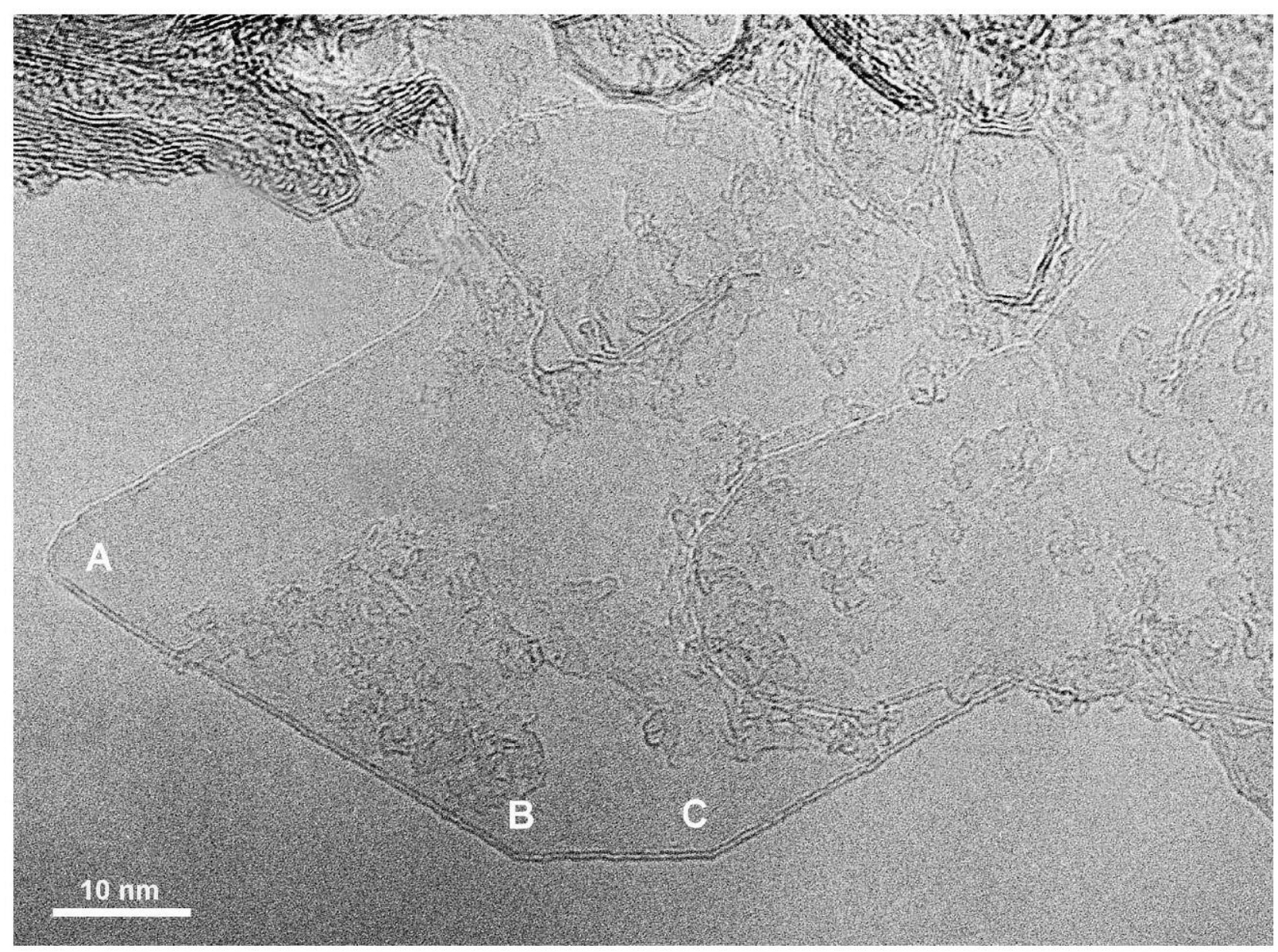

Figure 2 (Harris Fig 2 Revised.jpg) 

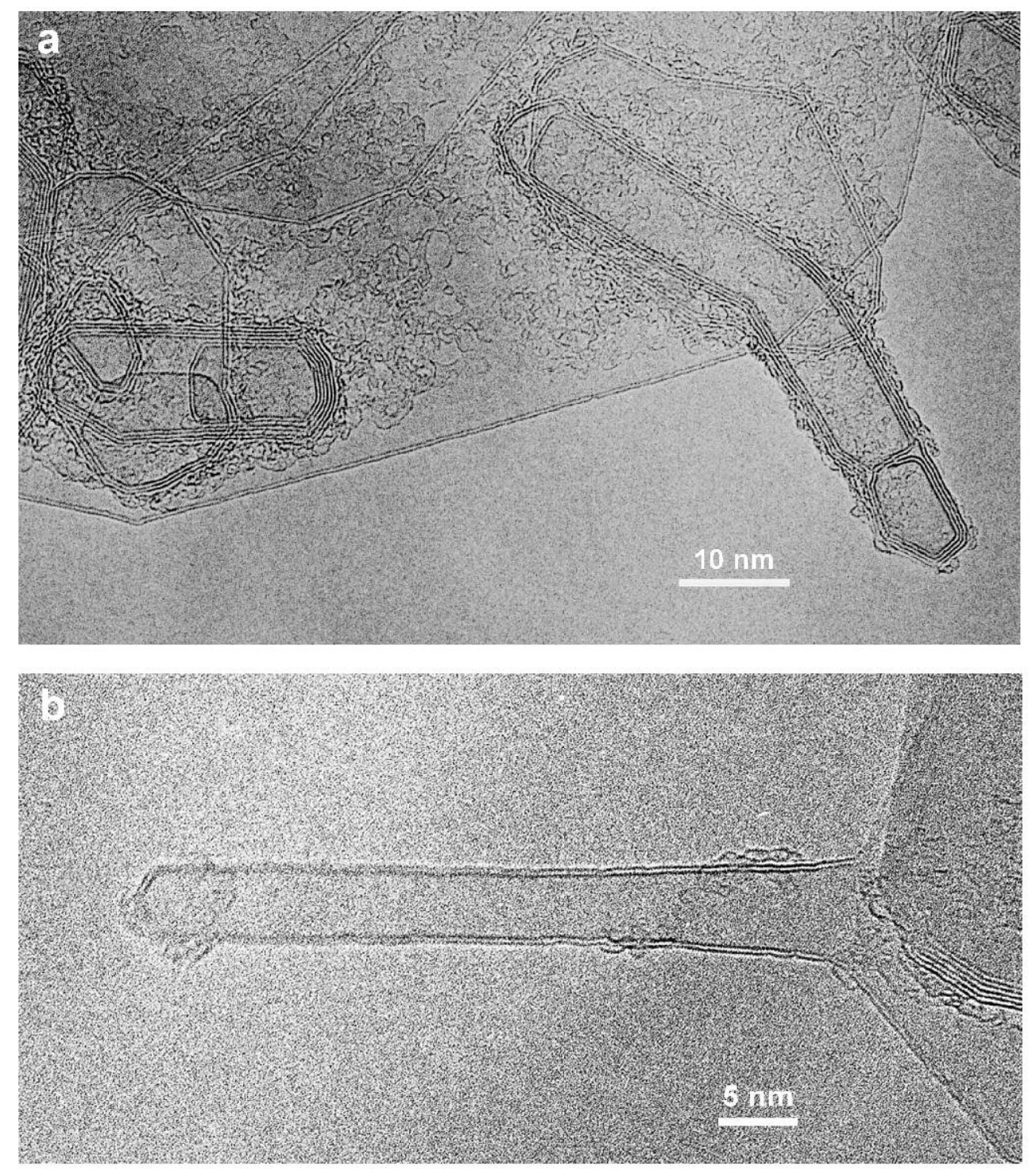

Figure 3 (Harris Fig 3.jpg) 

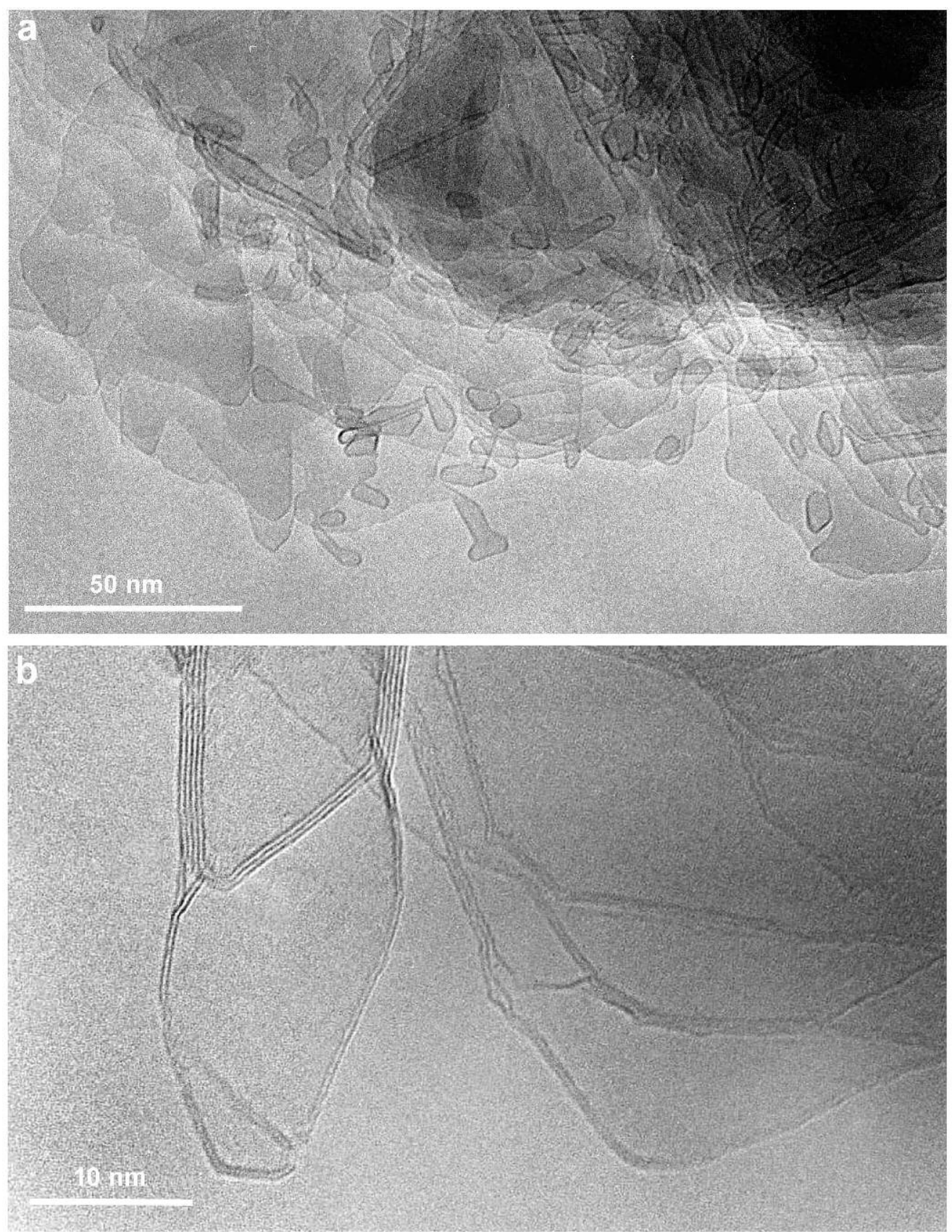

Figure 4 (Harris Fig 4.jpg) 